\title{
Advanced Simulation of Polycrystalline Electron Diffraction Patterns
}

\author{
X.Z. Li
}

Nebraska Center for Materials and Nanoscience, University of Nebraska, Lincoln, NE 68588

For polycrystalline TEM specimens, including fine powders and particles, electron diffraction ring patterns are extensively used for phase identification, in which the diffraction pattern of a known phase acts as a fingerprint [1]. A computer program, JECP/ PCED [2], was designed to provide a fast and accurate method for the task. The need for quantitative or semi-quantitative analysis of polycrystalline samples (powders and particles) requires the extension of the functions of the previous program. An upgraded version of the program, PCED2.0, has been developed to meet the requirement for phase identification, semi-quantitative analysis of structures and phases, and texture analysis and phase fraction determination [3].

Quantitative analysis of polycrystalline diffraction patterns consists of two basic steps: (i) digital processing of the experimental pattern to extract the quantitative data, and (ii) advanced simulation to interpret the data for phase analysis and/or structure determination and refinement. The PCED2.0 is developed for advanced simulation of polycrystalline electron diffraction patterns. New features in the PCED2.0 include: (i) Blackman two-beam dynamical correction of polycrystalline electron diffraction [4], (ii) March model for out-of-plane and in-plane texture [5], (iii) pseudo-Voigt peak profile fitting of diffraction rings, and (iv) the improved user-friendly interface in the handling of experimental diffraction data and the flexibility of indexing.

Formulae for polycrystalline electron diffraction intensities derived by Blackman [4] are based on Bloch wave dynamical theory using a two-beam approximation. The formulae have been proved to be successful in later experimental work and extended to the structural analysis of polycrystalline phases [6]. Some important equations are reformulated in Ref. [3] using the conventions in the book by Spence and Zuo [7]. In thin film samples, polycrystalline microstructures with out-of-plane or inplane preferred orientations often developed during film growth. The March model, originally formulated for X-ray powder diffraction (Bragg-Brentano geometry) in the paper by Dollase [5], can be slightly modified to apply to the electron diffraction intensities of the textured polycrystalline films by using selected-area electron diffraction geometry.

Figure 1 shows the GUI of the PCED2.0, which includes a drop-down menu, a toolbar, and a panel to show the results of the simulation. The menu and toolbar can be used to initiate dialog boxes for loading data or editing parameters. The screen shot in Figure 1 is a simulated diffraction pattern of two phases, f.c.c. $\mathrm{Al}$ and hexagonal $\mathrm{Mg}$. The peak profile of the diffraction rings from $\mathrm{Al}$ and $\mathrm{Mg}$ phases together with an incident beam is shown above the peak lines. A table shows the crystalline phases and important parameters for the simulation.

The purpose of the PCED2.0 is twofold as a teaching aid and a research tool. The program package was written in Java ${ }^{\mathrm{TM}} 5$ (JDK1.5), and has been tested on the Microsoft Windows ${ }^{\mathrm{TM}} \mathrm{XP}$ and Vista operating systems. As a Java program, the PCED2.0 is expected to run on other platforms with the $\mathrm{Java}^{\mathrm{TM}}$ runtime environment installed. A fully functional demonstration version of the program and a user's manual are available on the author's website [8]. 
References

[1] C.E. Lyman and M.J. Carr, in Electron Diffraction Techniques, Vol. 2, ed., John M. Cowley, Oxford Science Publications, 1993.

[2] X.Z. Li, Ultramicroscopy 99 (2004) 257.

[3] X.Z. Li, Ultramicroscopy (2010) in press.

[4] M. Blackman, Proceedings of the Royal Society of London, Series A, Mathematical and Physical Sciences, 173 (1939) 68.

[5] W.A. Dollase, J. Appl. Cryst. 19 (1986) 367.

[6] K. Vainshtein, B.B. Zvyagain and A.S. Avilov, in Electron Diffraction Techniques, Vol.1, ed. J.

M. Cowley, Oxford Science Publications, 1992.

[7] J.C.H. Spence and J.M. Zuo, Electron Microdiffraction, Plenum Press, 1992.

[8] http://www.unl.edu/CMRAcfem/XZLI/programs.htm.

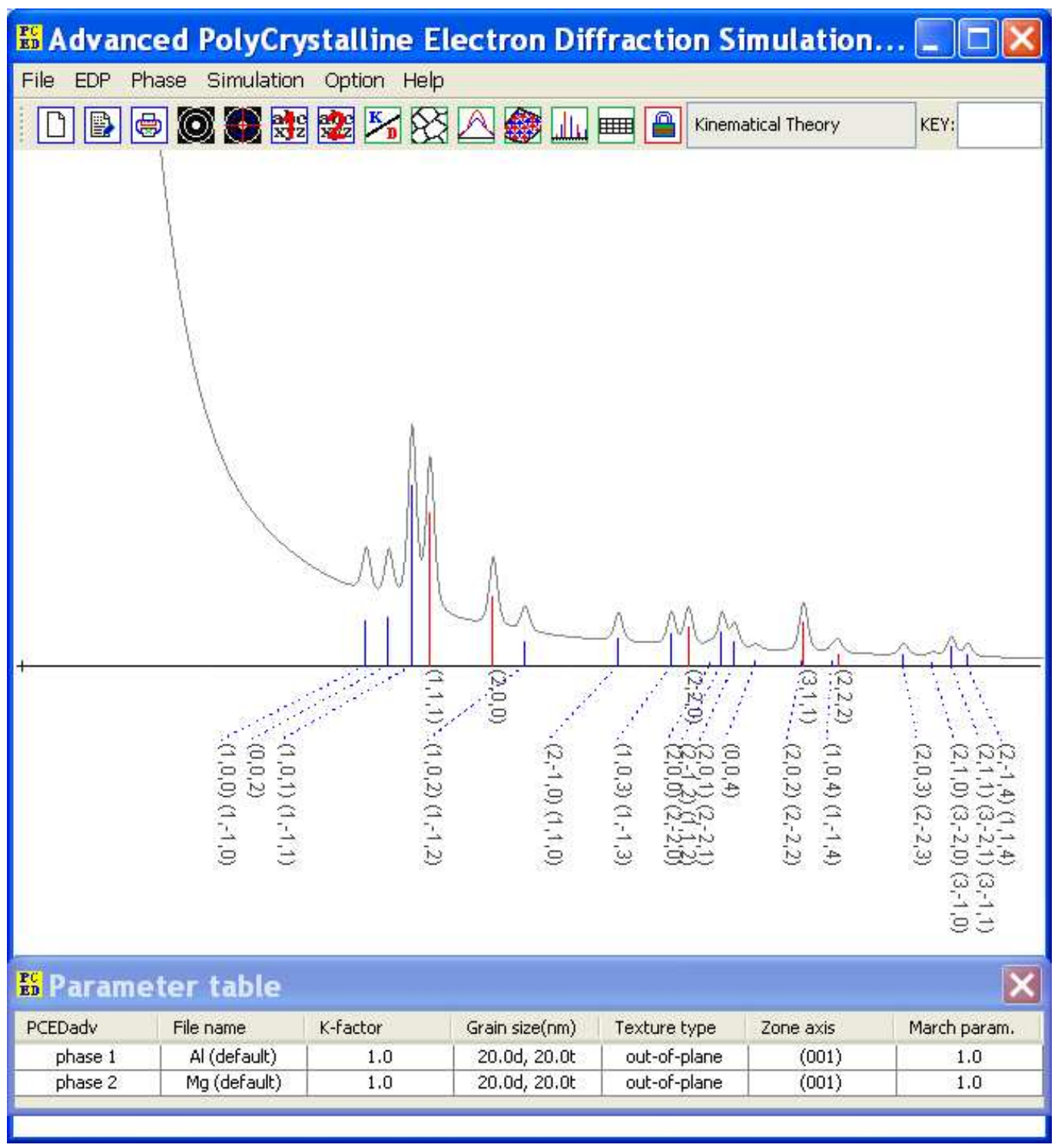

Fig. 1. Snap-shot of the graphical user interface of the PCED2.0. Simulated electron diffraction pattern of polycrystalline phases, fcc $\mathrm{Al}$ and hexagonal $\mathrm{Mg}$, are shown. 